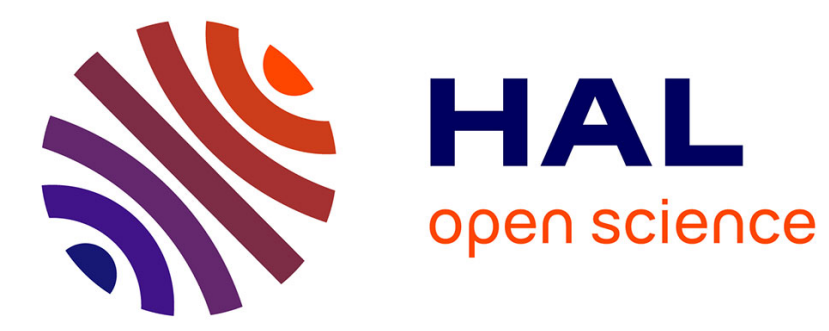

\title{
Theory and identification of a constitutive model of induced anisotropy by the Mullins effect
}

\author{
Guilherme Machado, Grégory Chagnon, Denis Favier
}

\section{To cite this version:}

Guilherme Machado, Grégory Chagnon, Denis Favier. Theory and identification of a constitutive model of induced anisotropy by the Mullins effect. Journal of the Mechanics and Physics of Solids, 2014, 63, pp.29-39. 10.1016/j.jmps.2013.10.008 . hal-00958608

\section{HAL Id: hal-00958608 \\ https://hal.science/hal-00958608}

Submitted on 12 Mar 2014

HAL is a multi-disciplinary open access archive for the deposit and dissemination of scientific research documents, whether they are published or not. The documents may come from teaching and research institutions in France or abroad, or from public or private research centers.
L'archive ouverte pluridisciplinaire HAL, est destinée au dépôt et à la diffusion de documents scientifiques de niveau recherche, publiés ou non, émanant des établissements d'enseignement et de recherche français ou étrangers, des laboratoires publics ou privés. 
6 Rubber-like materials present a stress softening phenomenon after a first loading known 7 as the Mullins effect. Some recent experimental data on filled silicone rubber is presented 8 in literature, using uniaxial and biaxial tests to precondition samples thus induce some 9 primary stress softening. A generic modeling based on the polymer network decomposition

*Corresponding author

Email address: gregory.chagnon@imag.fr (G. Chagnon) 
In a first approach, many isotropic models were proposed in the literature to describe stress softening. First, physical models taking into account the evolution of the chain network were proposed. Govindjee and Simo (1991) proposed a model based on the macromolecular network evolution by decomposition into a hyperelastic network and an evolving network. Marckmann et al. (2002) considered that the macromolecular network can be represented by the eight chains model (Arruda and Boyce, 1993). The model containing chain lengths and chain densities evolving with the maximal principal stretch. In another way, double network theory was developed (Green and Tobolsky, 1946) considering that the rubber-like material can be decomposed into a hard and a soft phase; the hard phase is transformed into soft phase with the stress softening. Different equations were proposed (Beatty and Krishnaswamy, 2000; Zuñiga and Beatty, 2002). At the same time, the damage theory was often used to describe the stress softening (Simo, 1987; Miehe, 1995; Chagnon et al., 2004). In another way, Li et al. (2008) associated the Mullins effect to the growth of cavities in the material and a compressible model was proposed. In a last point of view, Ogden and Roxburgh (1999) and Dorfmann and Ogden (2003) proposed models based on pseudo-elasticity. All these models fit experimental data more or less accurately in one loading direction, i.e., without changing loading direction between first and second loadings. For a more exhaustive review about these isotropic models, the reader can refer to Diani et al. (2009).

To improve the modeling and to fit anisotropic stress softening, new approaches were developed taking into account the difference of stress softening in each strain direction. At first, Göktepe and Miehe (2005) generalized the approach proposed by Govindjee and Simo (1991) taking into account a spatial repartition of the chains. In the same way, Diani et al. (2006a) proposed a generalization of the Marckmann et al. (2002) model by means of chains oriented into 42 or more directions in space. Using a phenomenological damage function, this model can describe different stress softening in different directions, with permanent deformation after unloading. Dargazany and Itskov (2009) proposed a similar approach by taking into account the existence of different chains with different lengths in each direction. They integrate the density of probability in each direction, by taking into account the network evolution at each step. Shariff (2006) proposed an anisotropic damage model that describes transverse anisotropy of Mullins effect, taking into account different damages in the three principal strain directions using a second-order 
damage tensor. In the same way, Itskov et al. (2010) proposed three damage evolution functions for the three principal strain directions. These functions are formulated in terms of material parameters that partly depend on the maximal principal stretch. Recently, Dorfmann and Pancheri (2012) proposed a phenomenological model, based on the theory of pseudo-elasticity, which includes scalar variables in the strain energy function to account for stress softening and changes in material symmetry.

Most of the anisotropic models mentioned above are proposed by analyzing successive tensile tests performed along different directions. In spite of that, Machado et al. (2012a) has recently performed other original tests based on preconditioning with uniaxial tension and biaxial tension tests. Based on Machado et al. (2012a) experimental results using silicone rubber, this paper proposes a new approach for modeling the induced anisotropy by the Mullins effect. In Section 2, the global framework of the Mullins effect modeling is presented. In Section 3, a new approach is proposed to write constitutive equations by introducing a tensor that describes the strain energy repartition in the space directions. The conditions to be verified by the equations are detailed. In Section 4 , a first constitutive equation is proposed. It is fitted and compared to experimental data. Finally, Section 5 contains some concluding remarks and outlines some future perspectives.

\section{Macromolecular approach to model Mullins effect}

\subsection{Filled silicone behavior}

In the last few years, different tests highlighting the stress softening anisotropy have been presented in the literature for different rubber-like materials, see for example (Muhr et al., 1999; Besdo et al., 2003; Hanson et al., 2005; Diani et al., 2006b; Dorfmann and Pancheri, 2012). In this paper, attention is focused on the largest and most diverse database concerning Mullins effect anisotropy of a rubber-like material to the best of our knowledge. These data concern the results for the RTV3428 filled silicone rubber (Machado et al., 2010, 2012a).

First classical experimental tests, i.e., cyclic experiments with an increasing deformation after each cycle, were realized during tensile, pure shear and equibiaxial tensile tests. The data are reported in Machado et al. (2010). Second, stress softening anisotropy is presented in Machado et al. (2012a) induced by two distinguished preconditioning methods. The first one (noted as TT in the following), consists in a first loading in tension and 
a second loading also in tension, in four different directions. The second preconditioning method (noted as BT), consists in a first biaxial extension loading with constant principal strain direction. It is followed by a second loading in tension along the two principal strain directions of the first biaxial loading. The originality of these data is that loading states are very different between the first and second loads.

These new experimental results question the existing anisotropic constitutive equations and the main reasons are detailed here. The first reason is, Diani et al. (2006a) and Dargazany and Itskov (2009) models present an important permanent deformation that is related to the stress softening. But here, the material exhibits an important stress softening without permanent deformation. The other reason, for a second tensile loading orthogonal to the first loading, the models of Shariff (2006) and Itskov et al. (2010) present a stiffer behavior than the virgin material, which is not the case of the filled silicone rubber. Last, all these models are based on a set of material directions and Mullins effect is controlled in each direction only by the maximum stretch reached during the deformation history along the considered direction. Recently, Merckel et al. (2011) analyzed the damage spatial repartition and proposed a softening criterion (Merckel et al., 2012) that is still the maximum stretch in each direction. Therefore, as pointed out in Machado et al. (2012a), a maximal deformation criterion that depends only on the considered direction is not enough to describe the stress softening for an arbitrary second load direction. This means that, if the maximal principal direction remains the same during the first and second load cycles, the strain energy can be a measure to quantify the Mullins effect in this direction. In the other directions, a coupling effect exists between different directions and it influences the stress softening. Under these circumstances, a new way to handle Mullins effect should be proposed at the sight of Machado et al. (2012a) experimental data.

\subsection{Two networks theory}

The results presented using silicone rubber-like materials highlight that unfilled silicone rubbers do not present stress softening (Rey et al., 2013) whereas filled silicone rubbers (Machado et al., 2010) present an important one. For this silicone rubber material, it can be argued that the Mullins effect is principally due to the presence of filler in the material, which is not the case for every rubber-like material. In the light of these findings, a model based on Govindjee and Simo (1991) theory is proposed. The main feature retained from their theory is the additive split of the strain energy into two contributions, motivated 

direction.

(a)

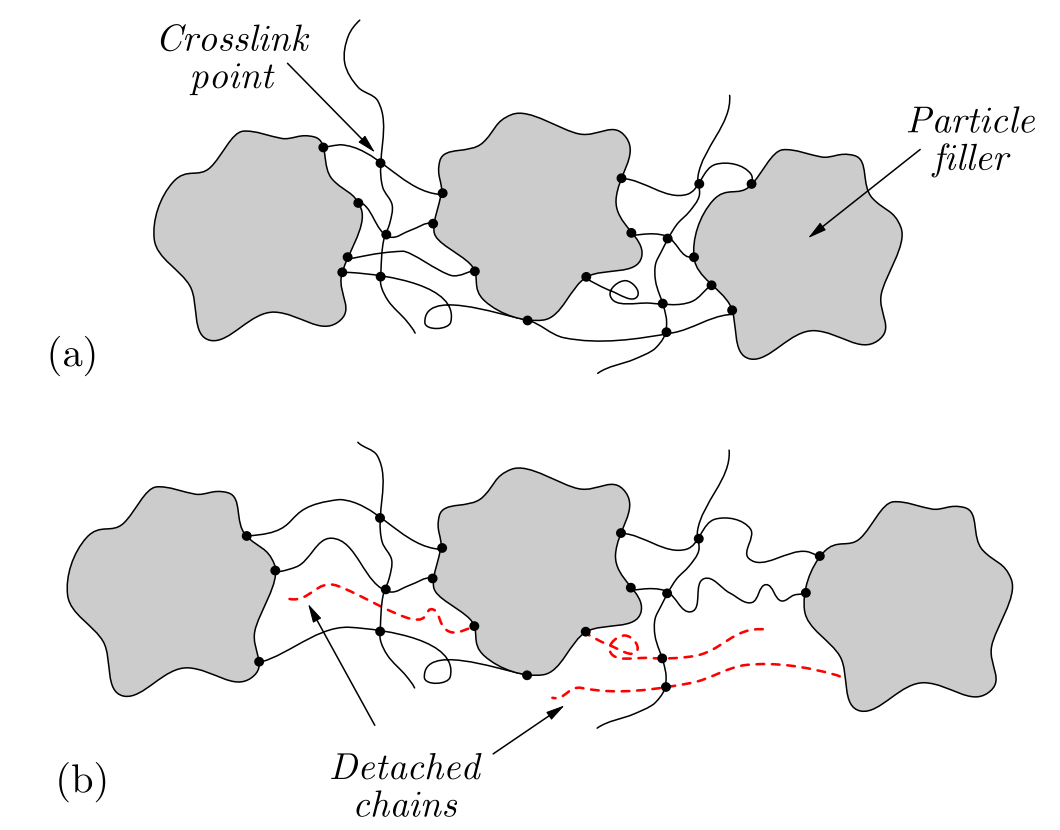

(b)

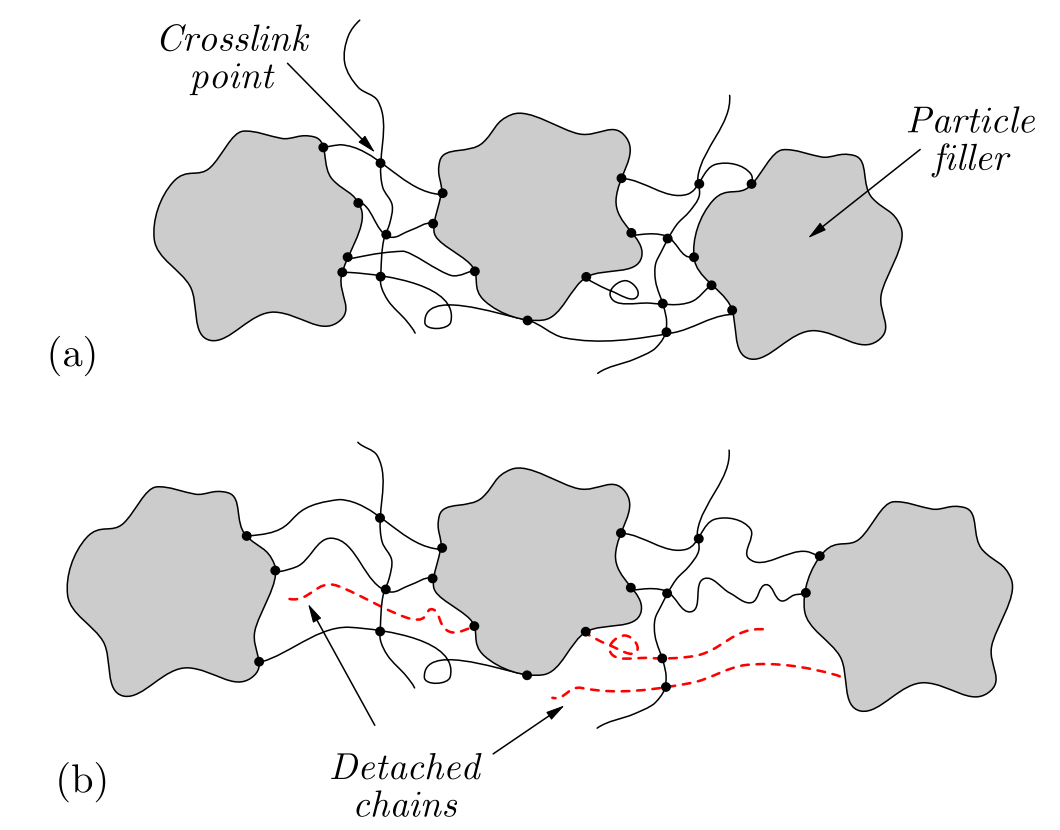

distinguishing different macromolecular chains in the network, those that are linked to filler and those that are only linked to other macromolecular chains. It is assumed that only chains that are linked to fillers are concerned with Mullins effect. In Govindjee and Simo (1992) authors modified the initial approach into a phenomenological isotropic frame, introducing a normalized stress function that governs the damage level. Later, Göktepe and Miehe (2005) conceptually extend the isotropic theory of Govindjee and Simo (1992) to the anisotropic case where damage history is described by one scalar for each material

Figure 1: Representation of the silicone organization with macromolecular chains and filler particles

The same additive split from Govindjee and Simo (1991) is considered here, that means that the strain energy density (per unit of undeformed volume) over a representative elementary volume (REV) is decomposed into two parts

$$
\mathcal{W}=\mathcal{W}_{c c}+\mathcal{W}_{c f}
$$

where $\mathcal{W}_{c f}$ and $\mathcal{W}_{c c}$ denote the energy densities of chains linked to filler network and chains linked to other chains network, respectively. On one hand, as it is considered that chains linked to other chains do not undergo Mullins effect, $\mathcal{W}_{c c}$ is therefore represented by an isotropic hyperelastic energy density. On the other hand, $\mathcal{W}_{c f}$ represents the anisotropy of stress softening induced by Mullins effect contributions in different directions of the REV. 


\section{Choice of the governing parameters of the Mullins effect}

\subsection{Analysis of literature experimental data}

The different conclusions identified by Machado et al. (2012a) are analyzed and the consequences for the modeling are here in detail. During TT tests (uniaxial tension preconditioning followed by second tensile tests) it is shown that whatever is the second loading orientation, the strain-stress curves come back on the first loading curve at the same point corresponding to the maximum tensile stretch encountered during the first tensile loading. Nevertheless the amount of stress softening depends on the angle between the first and second loadings. This means that the return on the tensile virgin curve is controlled by the maximal tensile stretch. However, the amount of stress softening depends on the relative orientation of first and second loadings.

The BT tests (biaxial tension followed by second tensile test) use preconditioning circular bulge test. Displacement and strain fields were obtained using three-dimensional image correlation measurements. In the preconditioning step, the circular bulge test specimen underwent biaxial loadings with different biaxiality ratios along a meridian. At the top of the bulge specimen, an equibiaxial loading is generated whereas a planar tension state is generated near the grips. Between these two points different biaxial states are generated (Machado et al., 2012b). For different points along the bulge meridian, pairs of specimens were cut along circumferential and meridional directions and they were tested in tension. For each pair, the two different second stress-strain tensile curves, in the same way of TT tests, come back at the same point on the virgin tensile loading curve but with a different amount of stress softening according to the direction (circumferential or meridional). The conclusions are thus the same as TT tests but with a biaxial loading as the preconditioning test.

These results encourage to consider that the strain energy in the maximal principal strain direction is the governing parameter for the come-back on the first loading curve whatever is the second loading. Moreover, the stress softening amount in the other directions are linked to this parameter but it is attenuated if the direction of the maximal principal strain is not the same between first and second loadings. A measure for these quantities should thus be introduced. 


$$
\hat{\mathbf{n}}=\operatorname{det}(\mathbf{F}) \frac{d S_{0}}{d S} \mathbf{F}^{-T} \mathbf{a}_{0} .
$$

The velocity field within the infinitesimal neighborhood of $\mathbf{x}$, with respect the reference

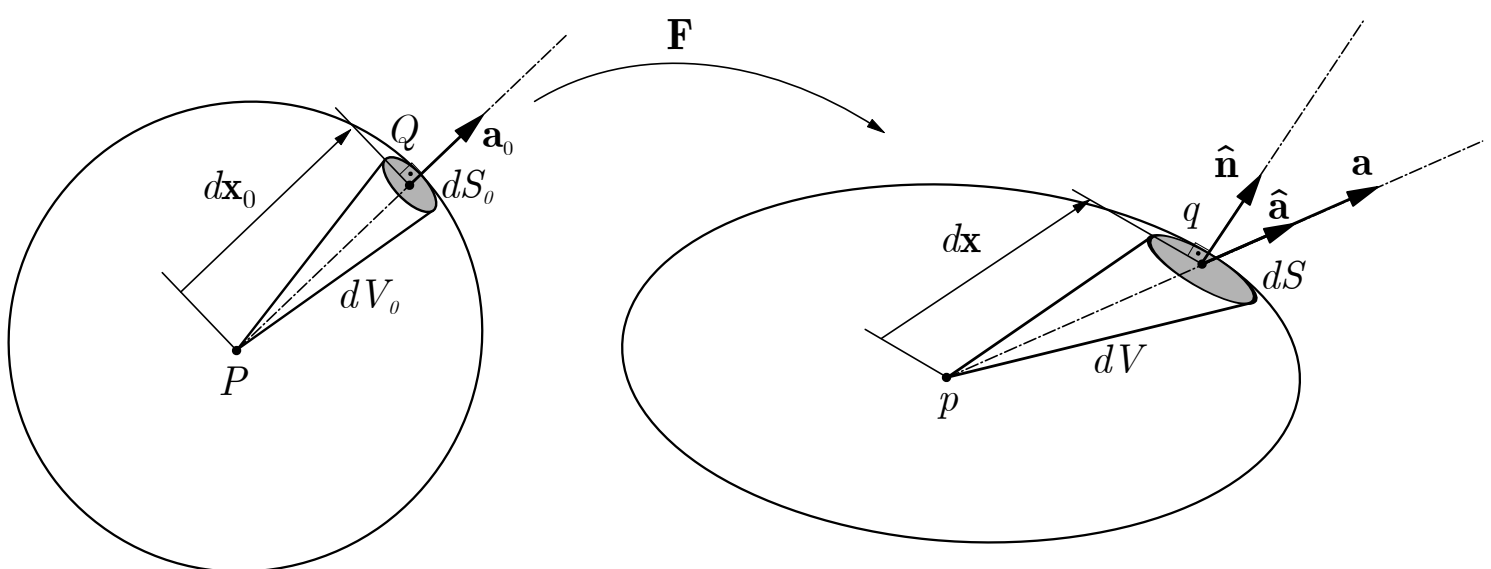

Figure 2: Kinematics of an infinitesimal cone element from the spherical REV. 


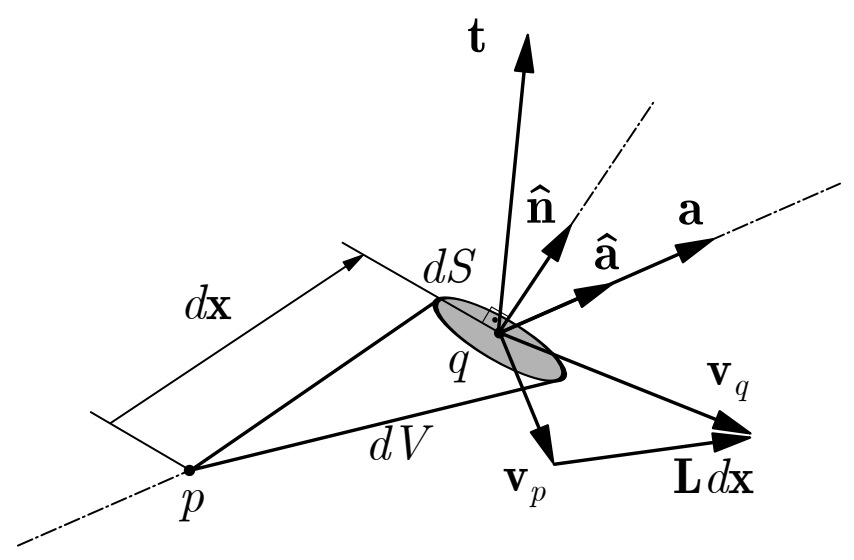

Figure 3: Force end velocity vectors in the deformed REV.

frame $R$ is given by

$$
\mathbf{v}_{q}(\mathbf{x}+d \mathbf{x})_{/ R}=\mathbf{v}_{p}(\mathbf{x}, t)_{/ R}+\mathbf{L}(\mathbf{x}, t)_{/ R} d \mathbf{x}
$$

with $\mathbf{L}(\mathbf{x}, t)_{/ R}=\mathbf{W}(\mathbf{x}, t)_{/ R}+\mathbf{D}(\mathbf{x}, t)$ where $\mathbf{L}, \mathbf{W}$ and $\mathbf{D}$ are the velocity gradient, spin and rate of deformation tensors. In any motion, the velocity field is locally decomposed as a sum of a rigid velocity $\mathbf{v}_{p}(\mathbf{x}, t)_{/ R}+\mathbf{W}(\mathbf{x}, t)_{/ R} d \mathbf{x}$ and a straining velocity $\mathbf{D}(\mathbf{x}, t) d \mathbf{x}$. Considering Figure 3, the power $\mathcal{P}_{/ R}=\mathbf{t} \cdot\left(\mathbf{v}_{q}\right)_{/ R}$ is expended by a force $\mathbf{t}=\boldsymbol{\sigma} \hat{\mathbf{n}} d S$ acting at point $q$, where $\boldsymbol{\sigma}$ is the Cauchy stress tensor. The interest is the expended power associated only with deformations. Then, it is possible to write the strain energy increment $d \mathcal{M}$ during a time increment $d t$, excluding rigid velocity, by the scalar product

$$
d \mathcal{M}=\boldsymbol{\sigma} \hat{\mathbf{n}} d S \cdot \mathbf{D} d \mathbf{x} d t
$$

where $\mathbf{D} d \mathbf{x}$ is the straining velocity field associated exclusively to the rate of deformation tensor D. Replacing Eq. (3) into Eq. (5), one obtains

$$
d \mathcal{M}=3 d V_{0} \operatorname{det}(\mathbf{F})\left[\mathbf{F}^{-1} \boldsymbol{\sigma} \mathbf{D F}\right]:\left(\mathbf{a}_{0} \otimes \mathbf{a}_{0}\right) d t
$$

Finally, the strain energy contribution in the $\mathbf{a}_{0}$ direction is written, per unity of undeformed volume $d V_{0}$, as

$$
\mathcal{M}\left(\mathbf{a}_{0}\right)=3\left[\int_{0}^{t} \operatorname{det}(\mathbf{F}) \mathbf{F}^{-1} \boldsymbol{\sigma D F} d t\right]:\left(\mathbf{a}_{0} \otimes \mathbf{a}_{0}\right)=3 \mathbf{M} \mathbf{a}_{0} \cdot \mathbf{a}_{0}
$$


tion. It is decomposed into a symmetric $\mathbf{M}^{\text {sym }}$ and skew-symmetric $\mathbf{M}^{\text {skew }}$ part. Note that the product of a symmetric tensor and a skew-symmetric tensor has zero trace, i.e., $\mathbf{M}^{\text {skew }}:\left(\mathbf{a}_{0} \otimes \mathbf{a}_{0}\right)=0$. Thus, in a general formulation, $\mathbf{M}^{\text {sym }}$ describes the contribution of each material direction in the total strain energy. As symmetric tensor $\mathbf{M}^{\text {sym }}$ possesses three real eigenvalues $\left(M_{I}>M_{I I}>M_{I I I}\right)$, where the maximal principal value $M_{I}$ is connected with an eigenvector determining the direction of maximum strain energy. Different noticeable parameters can be defined. The maximum strain energy for each conical elementary volume in direction $\mathbf{a}_{0}$ along the history is

$$
\mathcal{M}_{\max }\left(\mathbf{a}_{0}\right)=\max _{\tau \leq t} \mathcal{M}\left(\mathbf{a}_{0}, \tau\right)
$$

At the current time $t$, the maximum instantaneous strain energy in any direction is defined as

$$
\mathcal{I}(t)=M_{I}(t)
$$

And last, the maximum strain energy in any direction in the history is defined as

$$
\mathcal{G}=\max _{\tau \leq t} M_{I}(t)
$$

\subsection{Construction of the evolution equation}

An evolution function $\mathcal{F}$ is introduced along each direction, it describes the evolution of the network in the considered direction. The global strain energy is then rewritten as

$$
\mathcal{W}=\mathcal{W}_{c c}+\int_{V_{0}^{R E V}} \mathcal{F}\left(\mathbf{a}_{0}\right) \mathcal{W}_{c f}\left(\mathbf{a}_{0}\right) d V_{0}
$$

1 where $V_{0}^{R E V}$ is the undeformed REV volume. At the sight of the silicone filled rubber experimental data, the function $\mathcal{F}\left(\mathbf{a}_{0}\right)$ can be written as a function of the characteristic energy measures previously introduced

$$
\mathcal{F}\left(\mathbf{a}_{0}\right)=\mathcal{F}\left(\mathcal{M}\left(\mathbf{a}_{0}\right), \mathcal{M}_{\max }\left(\mathbf{a}_{0}\right), \mathcal{I}, \mathcal{G}\right)
$$

14 The main difference with the models from the literature is that the function $\mathcal{F}$ does not only depend on what happens in the considered direction $\mathbf{a}_{0}$ but also on the global strain energy in the material, i.e., $\mathcal{I}$ and $\mathcal{G}$. Then, different forms can be proposed. 

231 follows

$$
\mathcal{F}=1-\mathcal{F}_{1}(\mathcal{G}-\mathcal{I}) \quad \mathcal{F}_{2}\left(\mathcal{M}_{\max }\left(\mathbf{a}_{0}\right)-\mathcal{M}\left(\mathbf{a}_{0}\right)\right) \quad \mathcal{F}_{3}\left(\frac{\mathcal{M}_{\text {max }}\left(\mathbf{a}_{0}\right)}{\mathcal{G}}\right)
$$

232 where $\mathcal{F}_{1}, \mathcal{F}_{2}$ and $\mathcal{F}_{3}$ are functions to be determined. This multiplicative decomposition, 233 234 235 evoked in Eq. (13) lead to

$$
\begin{aligned}
\mathcal{F}_{1}(\mathcal{G}-\mathcal{I}) & =0 \quad \text { if } \quad \mathcal{G}=\mathcal{I} \\
\mathcal{F}_{2}\left(\mathcal{M}_{\max }\left(\mathbf{a}_{0}\right)-\mathcal{M}\left(\mathbf{a}_{0}\right)\right) & =0 \quad \text { if } \quad \mathcal{M}_{\max }\left(\mathbf{a}_{0}\right)=\mathcal{M}\left(\mathbf{a}_{0}\right) .
\end{aligned}
$$

${ }_{236}$ Now, different constitutive equation forms can be proposed for each function $\mathcal{F}_{1}, \mathcal{F}_{2}$ and ${ }_{237} \mathcal{F}_{3}$ in Eq. $(15)$.

237 . $F_{3}$ in $\mathrm{Eq}$. $(15)$. 


\section{The anisotropic constitutive equation}

\subsection{Hyperelastic constitutive equation}

The advantage of such formulation is that the first loading curve is independent of the evolution function on the contrary of damage mechanics (Lemaitre and Chaboche, 1990). Thus, the choice of the hyperelastic energy only depends on the first loading curves. In a first approach, it is proposed to use the classical Mooney (1940) constitutive equation to represent the isotropic energy density, then

$$
\mathcal{W}_{c c}=C_{1}\left(I_{1}-3\right)+C_{2}\left(I_{2}-3\right)
$$

For the anisotropic part of the constitutive equation the material could be represented by an infinite number of directions, introducing a probability density. But in this study, it is preferred to use a distribution of $n$-direction $\mathbf{a}_{0}^{(i)}$ oriented in any direction throughout the three-dimensional space instead of an integral formulation (Eq.11). Bazant and Oh (1986) proposed different orientation schemes, that define the set of vectors $\mathbf{a}_{0}^{(i)}$ with different weight $\omega^{(i)}$ for each direction to obtain a material as close as possible to an isotropic material when all the chains have the same mechanical behavior. $\mathcal{W}_{c f}$ is then written as

$$
\mathcal{W}_{c f}=\sum_{i=1}^{n} \omega^{(i)} \mathcal{F}^{(i)} \mathcal{W}_{c f}^{(i)}\left(\mathbf{a}_{0}^{(i)}\right)
$$

where $n$ is the number of considered directions and $\mathcal{W}_{c f}^{(i)}\left(\mathbf{a}_{0}^{(i)}\right)$ is the hyperelastic strain energy of the chain in the initial direction $\mathbf{a}_{0}^{(i)}$. The classical centrally symmetric $n=2 \times 21$ scheme was chosen to represent the material directions. The vector and weight of each direction can be found in Bazant and Oh (1986). All the other direction distribution schemes could also be used. A comparative study of recently proposed integration schemes in application to a full network model of rubber can be found in Ehret et al. (2010).

The non-Gaussian theory is classically used to capture the anisotropy. Diani et al. (2006a) and Dargazany and Itskov (2009) use the Langevin chain representation for $\mathcal{W}_{c f}^{(i)}$ energy. The great advantage of this choice is that it brings physical understanding to the modeling and it presents two main consequences. The first is that the zero-stress state is only ensured by the compensation of all the directions contribution as $\partial \mathcal{W}\left(\lambda^{(i)}\right) / \partial \lambda^{(i)} \neq 0$ if $\lambda^{(i)}=1$. Hence, this formulation could hardly be used for an initially non-isotropic 
material. The second one is that it allows to capture an important permanent deformation of the material after a loading cycle. However, in filled silicone experiments, it was shown that the permanent deformation is quite negligible. To this purpose, the classical hyperelastic anisotropic approach using the strain invariant $I_{4}^{(i)}=\mathbf{a}_{0}^{(i) T} \mathbf{C} \mathbf{a}_{0}^{(i)}$ is used, where $\mathbf{C}=\mathbf{F}^{T} \mathbf{F}$ is the right Cauchy-Green strain tensor. The function should verify the following conditions

$$
\begin{aligned}
\mathcal{W}_{c f}^{(i)}\left(I_{4}^{(i)}\right) & =0 \quad \text { if } \quad I_{4}^{(i)}=1 \\
\frac{\partial \mathcal{W}_{c f}^{(i)}\left(I_{4}^{(i)}\right)}{\partial I_{4}^{(i)}} & =0 \quad \text { if } \quad I_{4}^{(i)}=1 .
\end{aligned}
$$

In a first approach, an ordinary constitutive equation is used, considering that the chains are only stretched by tensile stresses. Otherwise, it is considered that compressive stretches lead to buckling. Thus, one may write

$$
\mathcal{W}_{c f}^{(i)}=\frac{K^{(i)}}{2}\left(I_{4}^{(i)}-1\right)^{2} \quad \text { if } \quad I_{4}^{(i)} \geq 1 \quad \text { else } \quad 0
$$

This formulation can be adapted to non-initially isotropic materials by choosing different functions for $\mathcal{W}_{c f}^{(i)}$. As the filled silicone rubber is initially isotropic, every $\mathcal{W}_{c f}^{(i)}$ is initially the same in all directions, i.e., $\forall i, j \quad K^{(i)}=K^{(j)}$.

\section{2. stress softening constitutive equation}

In part 3.3, a multiplicative decomposition was postulated in Eq. (15). The use of simple power functions, for $\mathcal{F}_{1}, \mathcal{F}_{2}$ and $\mathcal{F}_{3}$, is proposed to represent the stress softening, given by

$$
\mathcal{F}^{(i)}=1-\eta \sqrt{\frac{\mathcal{G}-\mathcal{I}}{\mathcal{G}}} \sqrt{\frac{\mathcal{M}_{\max }^{(i)}-\mathcal{M}^{(i)}}{\mathcal{G}}}\left(\frac{\mathcal{M}_{\max }^{(i)}}{\mathcal{G}}\right)^{2}
$$

where $\eta$ is a material parameter. The functions $\mathcal{F}_{1}$ and $\mathcal{F}_{2}$ are normalized according to the maximum strain energy $\mathcal{G}$ to ensure a normalized evolution function for each second loading curve. It is important to note that, even if the objective is to describe Mullins effect anisotropy, the constitutive equation for stress softening only depends on one parameter $\eta$. All the other parameters describe the hyperelastic first loading. The evolution functions have the same form in all directions, but this approach could be extended to non-isotropic stress softening function by defining different values for the parameter $\eta$ in the different 
directions.

There remains to verify that the presented model is in agreement with the requirements of thermodynamics (see e.g. Coleman and Gurtin, 1967). If only isothermal processes is considered, the Clausius-Duhem inequality must be satisfied by the conditions

$$
\begin{aligned}
-\frac{\partial \mathcal{W}}{\partial \mathcal{M}_{\max }^{(i)}} \dot{\mathcal{M}}_{\max }^{(i)} & \geqslant 0 \\
-\frac{\partial \mathcal{W}}{\partial \mathcal{G}} \dot{\mathcal{G}} & \geqslant 0
\end{aligned}
$$

where $\dot{\mathcal{M}}_{\max }^{(i)} \geqslant 0$ and $\dot{\mathcal{G}} \geqslant 0$. By means of straightforward manipulations of Eqs. (11), (24) and (25) one can easily establish the above relations in terms of the evolution function $\mathcal{F}^{(i)}$. It is also important to show that

$$
\begin{aligned}
\frac{\partial \mathcal{F}^{(i)}}{\partial \mathcal{M}_{\max }^{(i)}} & \leqslant 0, \quad \forall i \\
\frac{\partial \mathcal{F}^{(i)}}{\partial \mathcal{G}} & \leqslant 0, \quad \forall i .
\end{aligned}
$$

Considering the form of Eq. (23), the explicit form for Eq. (26) is given by

$$
-\eta \sqrt{\frac{\mathcal{G}-\mathcal{I}}{\mathcal{G}}} \frac{1}{\mathcal{G}}\left[\frac{1}{2}\left(\frac{\mathcal{M}_{\max }^{(i)}-\mathcal{M}}{\mathcal{G}}\right)^{-\frac{1}{2}}\left(\frac{\mathcal{M}_{\max }^{(i)}}{\mathcal{G}}\right)^{2}+2\left(\frac{\mathcal{M}_{\max }^{(i)}}{\mathcal{G}}\right) \sqrt{\frac{\mathcal{M}_{\max }^{(i)}-\mathcal{M}}{\mathcal{G}}}\right] \leqslant 0, \quad \forall i .(28)
$$

First, an elementary study of the Eq.(28) shows that all fractions terms are positive.

Second, when the stress softening evolves, i.e., $\mathcal{G}$ increasing, the maximum instantaneous strain energy $\mathcal{I}$ is equal to the maximum $\mathcal{G}$. Thus, the function remains equal zero and the condition of Eq. (26) is automatically satisfied. In this way, the choice of $\mathcal{F}$ respects the conditions of Eqs.(26) and (27) and consequently the Clausius-Duhem inequality is satisfied.

\subsection{Comparison of the modeling with experimental data}

The model is fitted on all the experimental data presented in Machado et al. (2010, 2012a), i.e., tests where the principal stretch directions remain unchanged during first and second load or tests where the principal stretch directions are not necessarily the same during first and second loads. First, the parameters of the hyperelastic constitutive equations are fitted on the different first loading curves. Different parameters can be chosen according to the repartition of the strain energy between $\mathcal{W}_{c c}$ and $\mathcal{W}_{c f}$. 
Considering a second tensile loading immediately before the sample rupture, the most stress softening level is obtained and this state corresponds to the strain energy of chains that were not affected by the Mullins effect. Thus, to ensure a good balance between $\mathcal{W}_{c c}$ and $\mathcal{W}_{c f}$ the portion $\mathcal{W}_{c c}$, i.e., the Mooney model, is fitted on the beginning of the second tensile loading curve at the higher deformation achieved before rupture.

Next, the part of $\mathcal{W}_{c f}$ is fitted to complete the stress amount of the first loading curves. The fitted parameters are presented in Table 1.

Table 1: Values of the constitutive equation parameters

\begin{tabular}{ll}
\hline Parameter & Value \\
\hline$C_{1}$ & $0.05 \mathrm{MPa}$ \\
$C_{2}$ & $0.03 \mathrm{MPa}$ \\
$\forall i \quad K^{(i)}$ & $0.20 \mathrm{MPa}$ \\
$\eta$ & 1.0 \\
\hline
\end{tabular}

The last parameter that describes the stress softening is fitted on the second loading curves for all the tests, the value $\eta=1.0$ is obtained. The condition in Eq. (14) must be satisfied, and as explained the function $\mathcal{F}^{(i)}$ cannot be negative. If its softening is too large, i.e., $\mathcal{F}^{(i)}<0$, the value $\mathcal{F}^{(i)}=0$ is imposed. That means that in the considered direction a great number of chain-filler links were broken. In the second load, for the same direction, the suspended chains are no longer acting enough to impose a force on the macromolecular network, i.e., they do not contribute to the network entropic energy any more and their energy is thus lost (Dargazany and Itskov, 2009). This assumption is consistent with the two networks theory and justified for relative short chains. Note that for longer molecular chains bonded at different places to fillers this assumption can be relaxed, for example, to take into account permanent set.

The simulations of the cyclic uniaxial tensile, pure shear and equibiaxial tensile tests are presented in Fig. 4. Concerning the first load, it appears that the model describes adequately uniaxial and pure shear tests whereas equibiaxial tests are underestimated. This phenomenon is due to the hyperelastic equation and not to stress softening equation. As pointed out by Marckmann and Verron (2006) and Boyce and Arruda (2000), there are very few hyperelastic constitutive models able to simultaneously simulate the both multi-dimensional data with a unique set of material parameters. Concerning the cyclic behavior, the form of the stress softening for all tests is quite well described. For uniaxial 


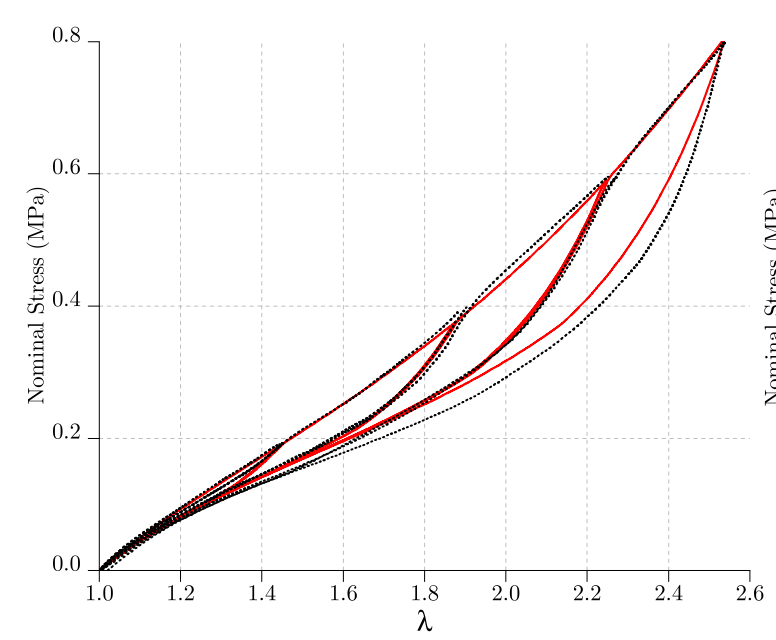

(a)

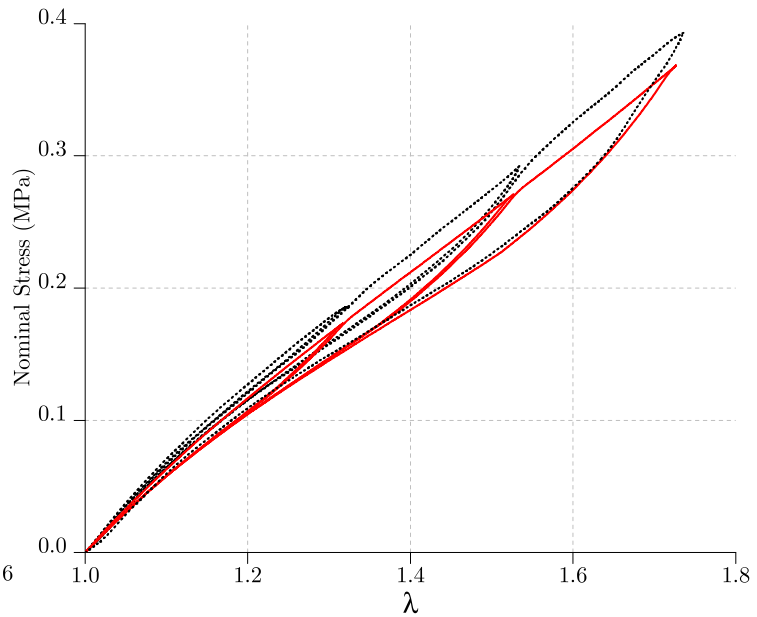

(b)

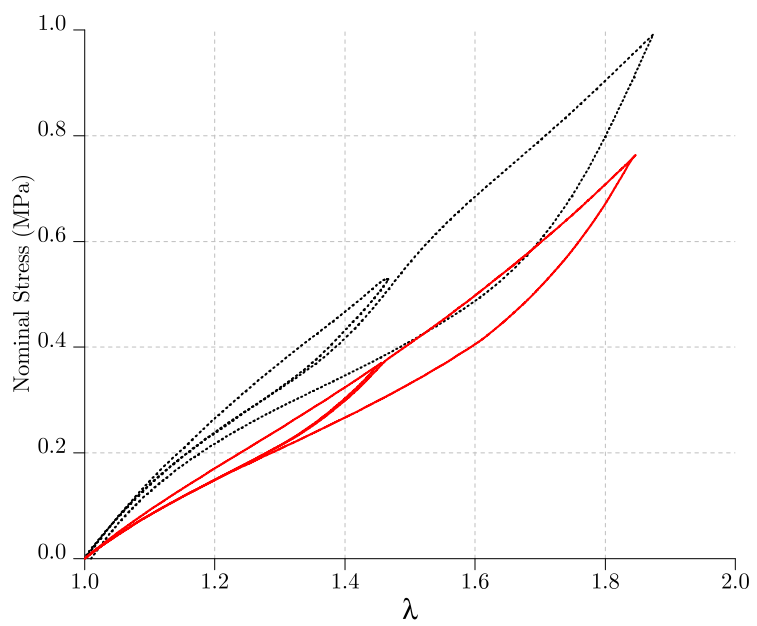

(c)

Figure 4: Comparison of the model (solid lines) with experimental data from Machado et al. (2010) (dotted lines) for: (a) cyclic uniaxial tensile test, (b) cyclic pure shear test and (c) cyclic equibiaxial test.

tensile and pure shear curves, the model has a slight tendency to underestimate stress softening and it is even more pronounced for equibiaxial tensile test. 


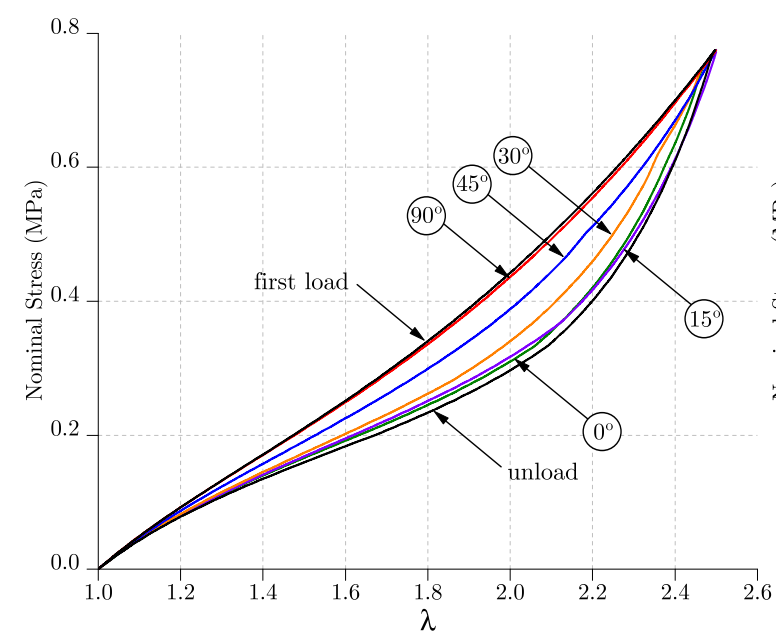

(a)

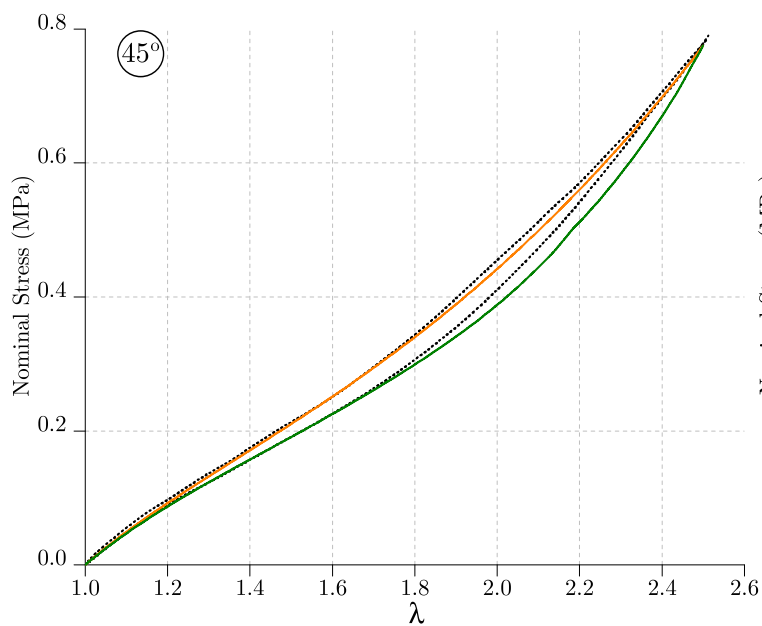

(c)

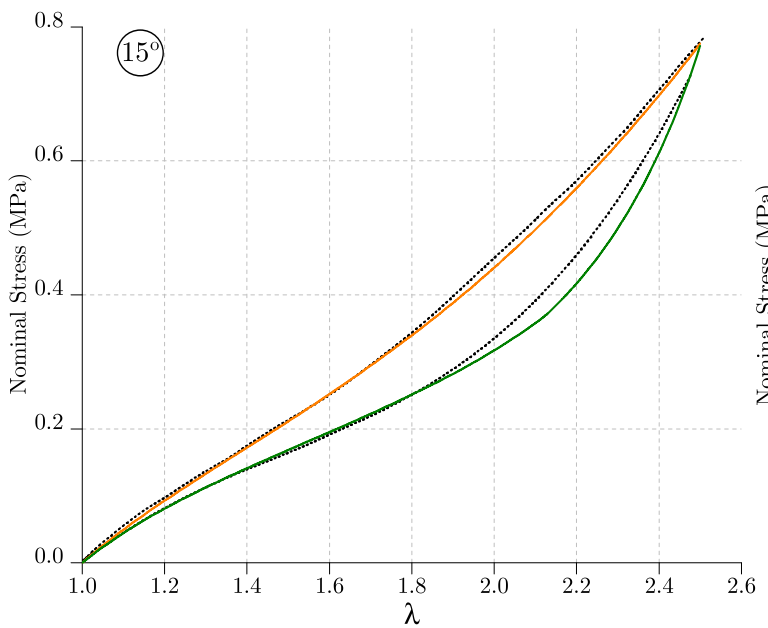

(e)

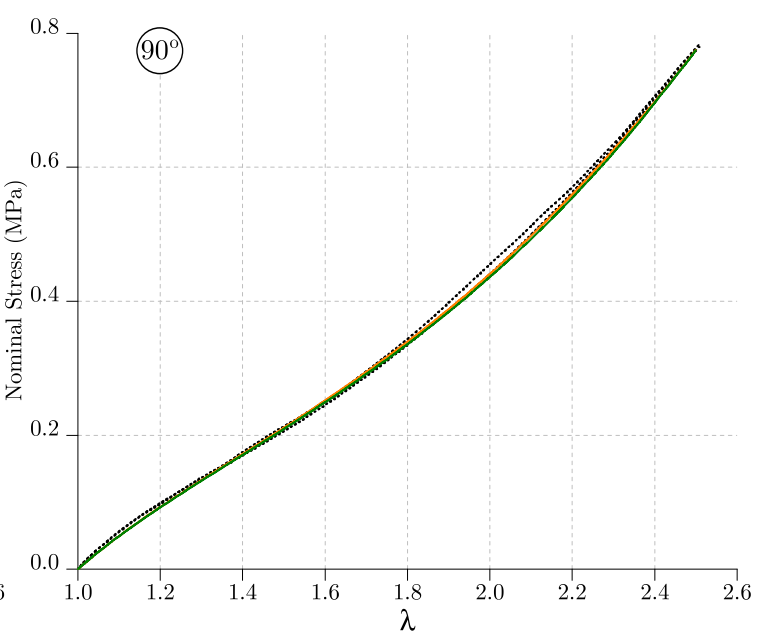

(b)

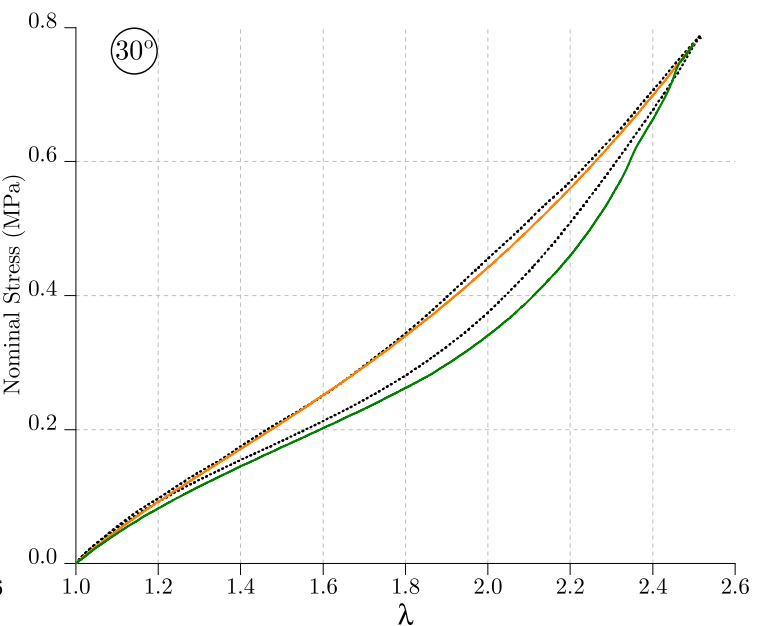

(d)

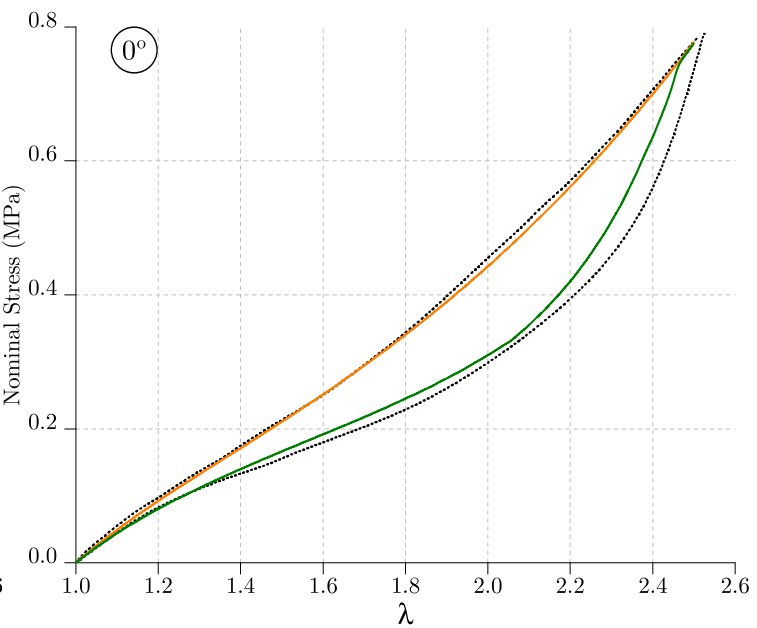

(f)

Figure 5: Comparison of the model (solid lines) with TT uniaxial prestretching experimental data (dotted lines). (a) simulation of the model for different orientations of the second load. Details of the experimental (dotted lines) and modeled (solid lines) first and second load curves with an angle between stretch direction of: (b) $90^{\circ}$, (c) $45^{\circ}$, (d) $30^{\circ}$, (e) $15^{\circ}$ and (f) $0^{\circ}$. 
To finish, the model is used to simulate BT tests, the first load being a biaxial test and the second load being a tensile test. The comparison of the second loading curves is presented in Fig. 6. It appears that stress softening is moderately overestimated by the model, however the come-back on the first loading curve is perfectly described.

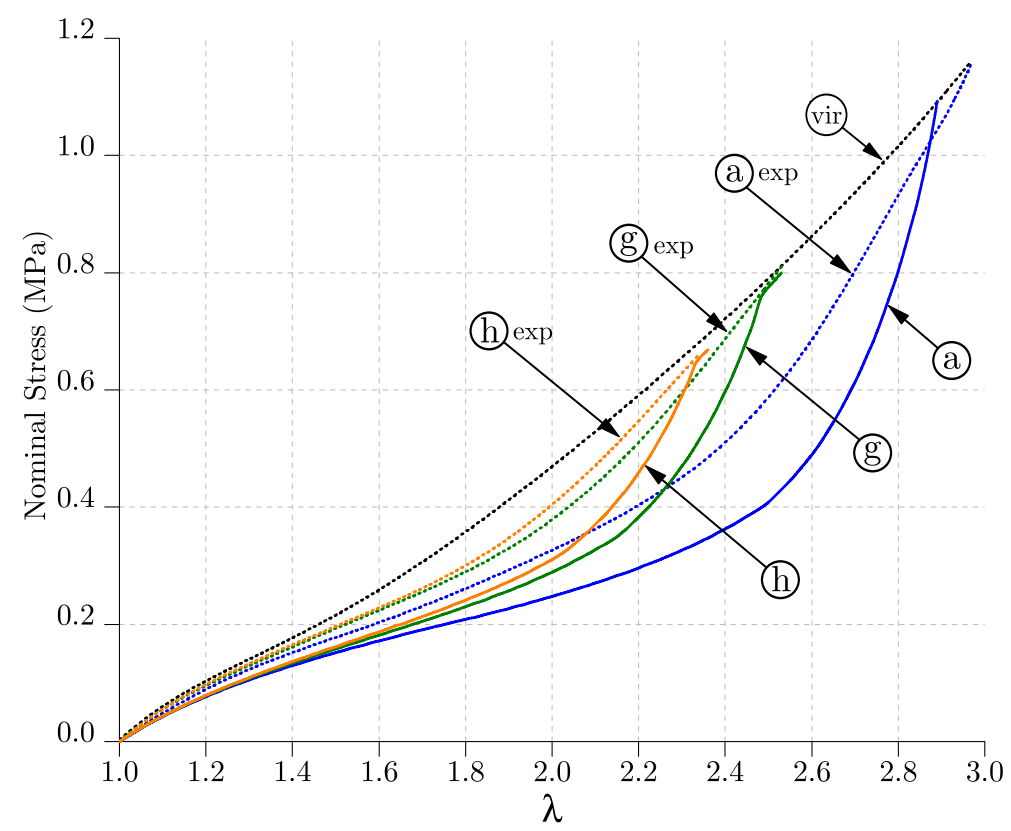

Figure 6: Comparison of the model (solid lines) with BT biaxial prestretching experimental data (dotted lines). Curve $a$ : simulation of the model for the second tensile load after an equibiaxial test; curve $g$ : simulation of the model for the second load after a biaxial test of biaxiality ratio $\mu=0.7$; curve $h$ : simulation of the model for the second load after a biaxial test of biaxiality ratio $\mu=0.5$

All these simulations emphasize that the use of this new elongation energy measure is a good point to describe the come back of the second loading curves on the virgin one. The amount of stress softening is well described for cyclic loading experiments and for TT tests, where the principal stretch directions were not the same during first and second loads. Nevertheless, the stress softening during BT test is overestimated. As observed in Fig. 4(c), stress was underestimated for the equibiaxial state, but this is due to the underestimation of hyperelastic strain energy obtained at the first load.

It can be noticed that the model describes correctly all the experimental tests with a simple constitutive equation that only depends on one parameter. Evidently, the results can be improved by proposing more complex constitutive equations, that consequently would lead to a significant increase in the number of parameters. Nevertheless, the presented results allowed to demonstrate the efficiency of this new approach. 


\section{Conclusion}

This paper presents an original approach to model the stress induced anisotropy by the Mullins effect, by the definition of a tensor to measure the repartition of the strain energy in space. The comparison of the strain energy in different directions with the maximal principal strain energy permits to create a new formulation for stress softening modeling. In this approach, the constitutive equation is written in function of the variation of strain energy in each direction and the variation of strain energy in the maximal principal strain direction. This new approach captures the principal characteristics of the Mullins effect underlined in literature. This new way of describing Mullins effect anisotropy can be a good starting point to elaborate new constitutive equations.

In this paper, a simple constitutive equation to describe the stress softening evolution was proposed. It clearly appears that the results are quite encouraging for a model that can describe many different types experimental tests, with very different strain histories, and the models presents only one material parameter. Of course, the agreement with the experimental data can be improved by using more sophisticated constitutive equation forms.

\section{Acknowledge}

We would like to thank the French ANR for supporting this work through the project RAAMO ("Robot Anguille Autonome pour Milieux Opaques")

\section{References}

Arruda, E. M. and Boyce, M. C. (1993). A three dimensional constitutive model for the large stretch behavior of rubber elastic materials. J. Mech. Phys. Solids, 41(2), 389-412.

Bazant, Z. P. and Oh, B. H. (1986). Efficient numerical integration on the surface of a sphere. Z. Angew. Math. Mech., 66, 37-49.

Beatty, M. F. and Krishnaswamy, S. (2000). A theory of stress-softening in incompressible isotropic materials. J. Mech. Phys. Solids, 48, 1931-1965.

Besdo, D., Ihlemann, J., Kingston, J., and Muhr, A. (2003). Modelling inelastic stress-strain phenomena and a scheme for efficient experimental characterization. In: Busfield, Muhr (eds) Constitutive models for Rubber III. Swets \& Zeitlinger, Lisse., pages 309-317.

Boyce, M. C. and Arruda, E. M. (2000). Constitutive models of rubber elasticity: A review. Rubber Chem. Technol., 73, 504-523. 
Chagnon, G., Verron, E., Gornet, L., Marckmann, G., and Charrier, P. (2004). On the relevance of Continuum Damage Mechanics as applied to the Mullins effect in elastomers. J. Mech. Phys. Solids, 52, 1627-1650.

Coleman, B. D. and Gurtin, M. E. (1967). Thermodynamics with internal state variables. J. Chem. Phys., $\mathbf{4 7}(2), 597-613$

Dargazany, R. and Itskov, M. (2009). A network evolution model for the anisotropic Mullins effect in carbon black filled rubbers. Inter. J. Solids Struct., 46(16), $2967-2977$.

Diani, J., Brieu, M., and Vacherand, J. M. (2006a). A damage directional constitutive model for the Mullins effect with permanent set and induced anisotropy. Eur. J. Mech. A/Solids, 25, 483-496.

Diani, J., Brieu, M., and Gilormini, P. (2006b). Observation and modeling of the anisotropic viscohyperelastic behavior of a rubberlike material. Int. J. Solids Struct., 43, 3044-3056.

Diani, J., Fayolle, B., and Gilormini, P. (2009). A review on the Mullins effect. Eur. Polym. Journal, 45, $601-612$.

Dorfmann, A. and Ogden, R. W. (2003). A pseudo-elastic model for loading, partial unloading and reloading of particle-reinforced rubbers. Int. J. Solids Struct., 40, 2699-2714.

Dorfmann, A. and Pancheri, F. (2012). A constitutive model for the Mullins effect with changes in material symmetry. International Journal of Non-Linear Mechanics, 47(8), $874-887$.

Ehret, A. E., Itskov, M., and Schmid, H. (2010). Numerical integration on the sphere and its effect on the material symmetry of constitutive equations - A comparative study. Int. J. Numer. Meth. Eng., 81(2), $189-206$.

Göktepe, S. and Miehe, C. (2005). A micro-macro approach to rubber-like materials. Part III: The microsphere model of anisotropic Mullins-type damage. J. Mech. Phys. Solids, 53, 2259-2283.

Govindjee, S. and Simo, J. C. (1991). A micro-mechanically continuum damage model for carbon black filled rubbers incorporating Mullins's effect. J. Mech. Phys. Solids, 39(1), 87-112.

Govindjee, S. and Simo, J. C. (1992). Transition from micro-mechanics to computationally efficient phenomenology: Carbon black-filled rubbers incorporating Mullins's effect. J. Mech. Phys. Solids, 40(1), 213-233.

Green, M. S. and Tobolsky, A. V. (1946). A new approach for the theory of relaxing polymeric media. J. Chem. Phys., 14, 87-112.

Hanson, D. E., Hawley, M., Houlton, R., Chitanvis, K., Rae, P., Orler, E. B., and Wrobleski, D. A. (2005). Stress softening experiments in silica-filled polydimethylsiloxane provide insight into a mechanism for the Mullins effect. Polymer, 46(24), 10989 - 10995.

Itskov, M., Ehret, A., Kazakeviciute-Makovska, R., and Weinhold, G. (2010). A thermodynamically consistent phenomenological model of the anisotropic Mullins effect. J. Appl. Math. Mech., 90(5), 370-386.

Lemaitre, J. and Chaboche, J. L. (1990). Mechanics of solid materials. Cambridge University Press.

Li, J., Mayau, D., and Lagarrigue, V. (2008). A constitutive model dealing with damage due to cavity growth and the Mullins effect in rubber-like materials under triaxial loading. J. Mech. Phys. Solids, 56(3), $953-973$. 
Machado, G., Chagnon, G., and Favier, D. (2010). Analysis of the isotropic models of the Mullins effect based on filled silicone rubber experimental results. Mech. Mater., 42(9), $841-851$.

Machado, G., Chagnon, G., and Favier, D. (2012a). Induced anisotropy by the Mullins effect in filled silicone rubber. Mech. Mater., 50, $70-80$.

Machado, G., Favier, D., and Chagnon, G. (2012b). Membrane curvatures and stress-strain full fields of axisymmetric bulge tests from 3D-DIC measurements. Theory and validation on virtual and experimental results. Exp. Mech., 52, 865-880.

Marckmann, G. and Verron, E. (2006). Comparison of hyperelastic models for rubber-like materials. Rubber Chem. Technol., 79(5), 835-858.

Marckmann, G., Verron, E., Gornet, L., Chagnon, G., and Fort, P. C. P. (2002). A theory of network alteration for the Mullins effect. J. Mech. Phys. Solids., 50, 2011-2028.

Merckel, Y., Diani, J., Roux, S., and Brieu, M. (2011). A simple framework for full-network hyperelasticity and anisotropic damage. J. Mech. Phys. Solids., 59(1), $75-88$.

Merckel, Y., Brieu, M., Diani, J., and Caillard, J. (2012). A Mullins softening criterion for general loading conditions. J. Mech. Phys. Solids., 60(7), $1257-1264$.

Miehe, C. (1995). Discontinuous and continuous damage evolution in Ogden type large strain elastic materials. Eur. J. Mech., A/Solids, 14(5), 697-720.

Mooney, M. (1940). A theory of large elastic deformation. J. Appl. Phys., 11, 582-592.

Muhr, A. H., Gough, J., and Gregory, I. H. (1999). Experimental determination of model for liquid silicone rubber: Hyperelasticity and Mullins effect. In Proceedings of the First European Conference on Constitutive Models for Rubber, pages 181-187. Dorfmann A. Muhr A.

Mullins, L. (1947). Effect of stretching on the properties of rubber. J. Rubber Res., 16, 275-289.

Ogden, R. W. and Roxburgh, D. G. (1999). A pseudo-elastic model for the Mullins effect in filled rubber. Proc. R. Soc. Lond. A, 455, 2861-2877.

Rebouah, M., Machado, G., Chagnon, G., and Favier, D. (2013). Anisotropic mullins stress softening of a deformed silicone holey plate. Mechanics Research Communications, 49(0), 36 - 43.

Rey, T., Chagnon, G., Le Cam, J.-B., Favier, D.(2013). Influence of the temperature on the mechanical behaviour of filled and unfilled silicone rubbers. Polym. Test., 32, 492-501.

Shariff, M. H. B. M. (2006). An anisotropic model of the Mullins effect. J. Eng. Math., 56(4), 415-435.

Simo, J. C. (1987). On a fully three-dimensional finite-strain viscoelastic damage model: formulation and computational aspects. Comp. Meth. Appl. Mech. Engng, 60, 153-173.

Zúñiga, A. E. and Beatty, M. F. (2002). A new phenomenological model for stress-softening in elastomers. Z. Angew. Math. Phys., 53, 794-814.

Zúñiga, A. E. (2005). A phenomenological energy-based model to characterize stress-softening effect in elastomers. Polymer, 46, 3496-3506.

Zúñiga, A. E. and Rodríguez, C. (2010). A non-monotonous damage function to characterize stresssoftening effects with permanent set during inflation and deflation of rubber balloons. Int. J. Eng. Sci., 48(12), $1937-1943$. 